\title{
Super-NOvA: A Long-baseline Neutrino Experiment with Two Off-axis Detectors
}

\section{Sergio Palomares-Ruiz*}

Department of Physics and Astronomy,

Vanderbilt University, Nashville, TN 37235, USA

E-mail: sergio.palomares-ruiz@vanderbilt.edu

\begin{abstract}
We consider here the NOvA experiment configuration and propose to place a second off-axis detector, with a shorter baseline, such that the type of neutrino mass hierarchy could be determined, by exploiting matter effects, with only the neutrino run. We show that the determination of this parameter is free of degeneracies, provided the ratio $L / E$, where $L$ the baseline and $E$ is the neutrino energy, is the same for both detectors. We also compare this possibility with the case of the second detector placed at the second oscillation maximum.
\end{abstract}

International Europhysics Conference on High Energy Physics

July 21st - 27th 2005

Lisboa, Portugal

${ }^{*}$ Speaker. 
The existence of neutrino oscillations [1] plays a crucial role in our understanding of neutrino physics, as it implies non-zero neutrino masses and mixing. Among the different parameters in this picture, the the type of neutrino mass spectrum, i.e., $\operatorname{sgn}\left(\Delta m_{31}^{2}\right)$ is one of the present unknowns. Its determination is of fundamental importance for the progress in the studies of neutrino mixing and provided $\theta_{13}$ is large enough, it might be determined in long-baseline experiments, which, in general, have a single detector with the beam running in both neutrino and antineutrino modes. However, these measurements suffer from degeneracies in the neutrino parameter space.

Generically, a measurement of the probability of conversion for neutrinos and antineutrinos cannot uniquely determine the type of hierarchy in a single experiment. Contrary to the naïve expectation, it has been shown in Refs. [2,3] that the use of only a neutrino beam could resolve the type of hierarchy when two long-baseline experiments are combined under certain conditions, being the best combination the one with the same $L / E$ at both experiments. Differently from this approach, we present a scenario with only one experiment, which runs in the neutrino mode and uses two detectors at different distances and off-axis angles (see Refs. $[4,5]$ for details). It is well known that off-axis neutrino beams have a very narrow neutrino spectra, and their peak energy can be tuned by displacing the detector out of the main beam axis. In such a way, a single beam could do the job of two beams with different energies.

Thus, we study the use of two off-axis detectors in combination with the NuMI beam so that the type of hierarchy could be determined free of other degeneracies. We consider one detector at the location which is the most likely for the NOvA configuration $(L=810 \mathrm{~km}$ and $E=2.3 \mathrm{GeV})$ and the other at $L=200 \mathrm{~km}$. We name this improved experimental setup Super-NOvA. We show that a configuration with the same vacuum oscillation phase, i.e., same $L / E$ for both detectors, is specially sensitive to matter effects and hence to $\operatorname{sgn}\left(\Delta m_{31}^{2}\right)$. In addition, we show that such a measurement is free of degeneracies, which provides the full power of this method.

We consider the case of $L / E$ constant and show how, by adding another detector to the already proposed NOvA experimental setup, the measurement of $\operatorname{sgn}\left(\Delta m_{31}^{2}\right)$ is possible free of degeneracies from other parameters. We have explored the possibility of using two 50 kton liquid argon TPC detectors, for which the efficiency to identify $v_{e}$ CC interactions is $90 \%$ (i. e., basically perfect efficiency) and that the background is dominated by the intrinsic $v_{e}$ and $\bar{v}_{e}$ components of the beam. We have assumed that the number of protons on target per year is $3.7 \times 10^{20}[6]\left(18.5 \times 10^{20}\right.$ pot/yr with a proton driver) ${ }^{1}$ and five years of neutrino running. On the other hand, in order to be sensitive to $\mathrm{CP}$-violation, the running with antineutrinos is completely necessary, but we do not consider this possibility here (see Ref. [5] for a detailed study of this possibility with different choices of detectors and scenarios).

In order to study matter effects, we consider the probability of $v_{\mu}-v_{e}$ oscillations in matter at two different lengths of the baselines, $L_{\mathrm{N}}$ (near detector) and $L_{\mathrm{F}}$ (far detector), so that we keep the same ratio $L / E$ at both sites. By calculating the normalized difference of the oscillation probabilities $(\mathscr{D})$ computed at the near and far locations, we can see that its sign depends upon the type of neutrino mass spectrum [4], and that the effects due to the CP-phase $\delta$, as well as those due to the solar neutrino mixing parameters, are subleading and cannot flip the sign of $\mathscr{D}$ for large enough values of $\sin ^{2} 2 \theta_{13} \geq 0.01$, which are within the range to be explored by the NOvA experiment $[6,7]$.

\footnotetext{
${ }^{1}$ These values correspond to the already old NOvA proposal [6]. For the recent revised proposal see Ref. [7].
} 


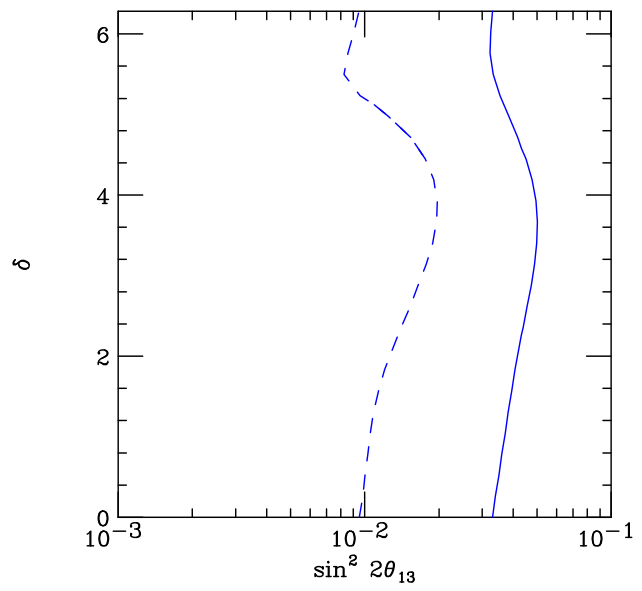

(a)

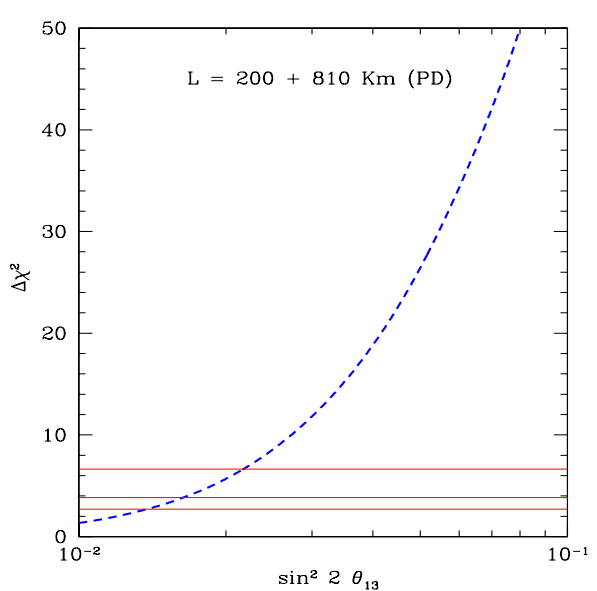

(b)

Figure 1: (a) Sensitivity to $\operatorname{sgn}\left(\Delta m_{31}^{2}\right)$ (for $\left|\Delta m_{31}^{2}\right|=2.4 \times 10^{-3} \mathrm{eV}^{2}$ ), including systematic errors. The solid (dashed) curve is obtained without (with) proton driver. (b) Results of the $\chi^{2}$ analysis to determine $\operatorname{sgn}\left(\Delta m_{31}^{2}\right)$ versus $\sin ^{2} 2 \theta_{13}$ with a proton driver. The corresponding $90 \%, 95 \%$ and $99 \%$ C.L.s are shown. See text regarding the experimental setup. From Ref. [4].

In order to compute the sensitivity to the mass hierarchy, as a first approach, we have constructed a measurable integrated asymmetry:

$$
A_{+}=\frac{\left\{N / N_{o}\right\}_{\mathrm{N}}-\left\{N / N_{o}\right\}_{\mathrm{F}}}{\left\{N / N_{o}\right\}_{\mathrm{N}}+\left\{N / N_{o}\right\}_{\mathrm{F}}},
$$

where $N$ is the number of $v_{e}$ induced events in the presence of oscillations and $N_{o}$ is the expected number of $v_{\mu}$ charged-current interactions in the absence of oscillations at the near (N) and far (F) detectors. We present the $95 \%$ C.L. sensitivities to the neutrino mass hierarchy resolution in Fig. 1a [4], where Nature's solution has been chosen to be the normal hierarchy.

We have also performed an independent $\chi^{2}$ analysis on the $\sin ^{2} 2 \theta_{13}-\delta$ plane, where we have assumed that Nature has chosen a given sign for $\Delta m_{31}^{2}$, but the analysis is performed with the opposite sign [4]. We show these results in Fig. 1b, where we plot the sensitivity to $\operatorname{sgn}\left(\Delta m_{31}^{2}\right)$ for the case with a proton driver. From these studies, we observe that the type of hierarchy could be determined regardless of the value of the CP-phase $\delta$ down to values of $\sin ^{2} 2 \theta_{13}=0.04$ or $\sin ^{2} 2 \theta_{13}=0.02$ with a proton driver at $95 \% \mathrm{CL}$.

Interestingly, in the revised NOvA proposal [7], the possibility to add a second detector is pointed out. The approach there consists in placing the second detector at the second oscillation maximum, where the matter effect is smaller by a factor of three because the energy is smaller by the same factor. However, for such a configuration there is no cancellation between the vacuum terms at both detectors, and the $\mathrm{CP}$-violating effects are also different (larger by a factor of three in the second detector for the same reason as above). These facts imply that the matter-dependent terms are of the same order of those carrying information on $\mathrm{CP}$-violation, which makes much more difficult to disentangle the type of mass hierarchy from $\mathrm{CP}$-violating effects. In Fig. 2, we compare these two possibilities for placing the second detector in the case of a proton driver. As was anticipated, the configuration for which both detectors are located such that the vacuum oscillation phase is the same (thick solid line), i.e., the same $L / E$, is much better when running only with neutrinos than that with the second detector at the second oscillation maximum (thin solid line). 


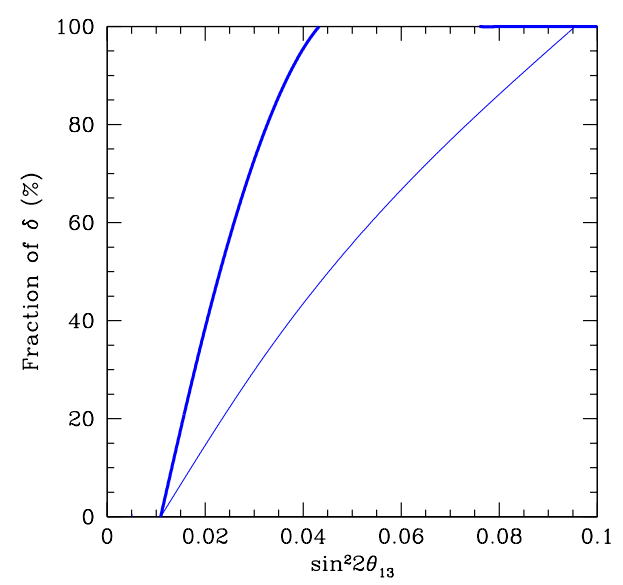

Figure 2: Comparison of the capabilities for determining $\operatorname{sgn}\left(\Delta m_{31}^{2}\right)$ at $95 \%$ C.L. for two different setups for the second detector: at the same $L / E$ as the far detector, as the proposal here (thick line) and at the second oscillation maximum, as the NOvA proposal (thin line). We have assumed the use of a proton driver. From Ref. [5].

In summary, we have presented a method for establishing $\operatorname{sgn}\left(\Delta m_{31}^{2}\right)$, free of degeneracies, by using one experiment running only in the neutrino mode. We have considered an experimental setup with two neutrino detectors placed in a special off-axis configuration. It is known that off-axis spectra are well peaked at a certain neutrino energy, which depends on the angle from the central axis of the beam. This allows both detectors to be located in such a way that they have the same $L / E$. We have shown that synergy effects show up with this special configuration for which vacuum oscillation phases are the same at both sites, stressing the difference in matter effects. This makes this configuration a better approach than that with the second detector at the second maximum.

\section{Acknowledgments}

These results were obtained in collaboration with O. Mena and S. Pascoli. SPR is supported by NASA Grant ATP02-0000-0151 and by the Spanish Grant FPA2002-00612 of the MCT.

\section{References}

[1] S. Fukuda et al. [Super-Kamiokande Collaboration], Phys. Lett. B 539, 179 (2002); S. N. Ahmed et al. [SNO Collaboration], Phys. Rev. Lett. 92, 181301 (2004); B. Aharmim et al. [SNO Collaboration], nucl-ex/0502021; Y. Ashie et al. [Super-Kamiokande Collaboration], Phys. Rev. D 71, 112005 (2005); T. Araki et al. [KamLAND Collaboration], Phys. Rev. Lett. 94, 081801 (2005); E. Aliu et al. [K2K Collaboration], Phys. Rev. Lett. 94, 081802 (2005).

[2] P. Huber, M. Lindner and W. Winter, Nucl. Phys. B 654, 3 (2003).

[3] H. Minakata, H. Nunokawa and S. J. Parke, Phys. Rev. D 68, 013010 (2003).

[4] O. Mena, S. Palomares-Ruiz and S. Pascoli, Phys. Rev. D 72, 053002 (2005).

[5] O. Mena, S. Palomares-Ruiz and S. Pascoli, hep-ph/0510182.

[6] I. Ambats et al. [NOvA Collaboration], FERMILAB-PROPOSAL-0929, March 15, 2004, available at http://www-nova.fnal.gov/reports_page.html

[7] D. S. Ayres et al. [NOvA Collaboration], hep-ex/0503053. FERMILAB-PROPOSAL-0929, March 21, 2005. Revised NOvA Proposal. 